\title{
Biomimetic auto-separation of leukocytes from whole blood in a microfluidic device
}

\author{
Sergey S. Shevkoplyas ${ }^{\dagger}$, Tatsuro Yoshida ${ }^{\dagger}$, Lance L. Munn ${ }^{*,+}$ and Mark W. Bitensky ${ }^{*,+}$
}

${ }^{\dagger}$ Visual and Circulatory Biophysics Laboratory, Department of Biomedical Engineering, Boston University; ${ }^{~}$ E. L. Steele Laboratory for Tumor Biology, Department of Radiation Oncology, Massachusetts General Hospital and Harvard Medical School.

\begin{abstract}
Movie S-1. The movie represents dynamics at the entry into the extraction channel. Leukocytes traveling in the plasma rich zone near the wall are collected into the $10-\mu \mathrm{m}$ wide extraction channel. Erythrocyte-leukocyte collisions, leading to leukocyte margination upstream, and plasma skimming contribute to the efficiency of separation.
\end{abstract}

